\title{
Los informantes (des)calificados. Análisis de una técnica de investigación cualitativa para niños que usan tecnología en la escuela
}

Datos de contacto:

Alicia Kachinovsky Melgar Universidad de la República, Uruguay

alika@psico.edu.uy

Michel Dibarboure Reynes Universidad de la República, Uruguay

mdibarboure@gmail.com

Joaquín Paredes-Labra Universidad Autónoma de

Madrid

joaquin.paredes@uam.es

Recibido: 29/04/2020

Aceptado: 26/01/2021

\author{
Alicia KACHINOVSKY MELGAR \\ Michel DIBARBOURE REYNES \\ Joaquín PAREDES-LABRA
}

\section{RESUMEN}

Profesores, estudiantes y centros educativos son objeto de caracterización para tratar de comprender cómo se alcanza la competencia digital, en qué se manifiesta y qué implicaciones tiene. El presente artículo aborda la frecuente ausencia del punto de vista de los niños como informantes calificados en investigaciones sobre infancia y TIC. Para justificar la inclusión de estas voces en el estudio de problemáticas que los afectan, se proponen razones epistemológicas, diferencias comunicacionales entre adultos y niños y derechos de infancia que condicionan una particular ética de investigación. Se pregunta por la adecuación de las herramientas para recabar la perspectiva infantil y se ilustran los recursos técnicos utilizados para afrontarlo. La metodología del estudio revisa una batería de estudios dentro de una investigación internacional que ha procurado incluir estas técnicas. En concreto, se analiza una variación de la técnica de la caja de juegos, que se sustituye por una computadora portátil, y la aportación de los diarios de los estudiantes en la caracterización de las prácticas con tecnología. Entre los resultados se observa que para fundamentar la especificidad del discurso infantil se requiere de técnicas específicas. Al respecto, las técnicas utilizadas permiten caracterizar la producción infantil con tecnología. Por otra parte, frente a teorizaciones por parte de los adultos sobre las producciones de los estudiantes, estas técnicas permiten superar el sesgo de interpretación que tiene un discurso centrado en el adulto. Es necesario, en cualquier caso, triangular los resultados con otras pruebas.

PALABRAS CLAVE: infancia; investigación cualitativa; dispositivos; discurso. 


\title{
(Un) qualified informants. Analysis of a qualitative research technique for children that use technology at school
}

\begin{abstract}
Teachers, students and schools are object of characterization to try to understand how digital competence is achieved, in what it manifests and what implications it has. This article addresses the frequent absence of children's point of view as qualified informants in childhood and ICT research. To justify the inclusion of these voices in the study of issues that affect them, epistemological reasons, communicational differences between adults and children, and children's rights that determine a particular research ethic are proposed. It is asked about the adequacy of the tools to obtain the child perspective and the technical resources used to face it are illustrated. The study methodology reviews a battery of studies within an international investigation that has attempted to include these techniques. Specifically, a variation of the game box technique, which is replaced by a laptop, and the contribution of student diaries in characterizing practices with technology are analyzed. Among the results, it is observed that specific techniques are required to support the specificity of children's speech. In this regard, the techniques used allow characterizing children's production with technology. On the other hand, in the face of theorizing by adults about student productions, these techniques allow to overcome the interpretation bias that an adult-centered discourse has. In any case, it is necessary to triangulate the results with other tests
\end{abstract}

KEYWORDS: childhood; qualitative research; devices; discourse. 


\section{Introducción}

El objetivo de este trabajo es valorar la necesidad de incluir a los niños en calidad de informantes calificados cuando se trata de problemas que les atañen directamente, por medio de técnicas específicas de recolección de datos. Tal es el caso cuando se procura examinar el uso e impacto de las TIC en la vida de los niños y su entorno. Al estudiar temas que comprometen su bienestar suele ignorarse el punto de vista de quienes son los más afectados, no tomando en cuenta su discurso. Se recogen concepciones de los adultos -padres, docentes, periodistas, políticossobre asuntos que aquejan a los niños. ¿Por qué no darles la voz a estos informantes calificados? Esto pone sobre la mesa principios epistemológicos, atributos del discurso infantil y una perspectiva de derechos, tres cuestiones que serán atendidas en el presente trabajo a la luz de las técnicas cualitativas que se pueden desarrollar para ello.

Algunos autores sostienen que se ha producido en los últimos años un cambio significativo en lo que hace a la investigación con niños, debido al reconocimiento de los derechos de infancia y a la reconceptualización de los niños por las ciencias sociales como agentes activos, no como meros objetos de investigación (Kirk, 2007). El creciente interés por incluirlos supone con frecuencia la utilización de materiales audiovisuales o audio-verbo-visuales junto a otras técnicas más tradicionales (entrevistas, grupos de discusión). La academia anglosajona parece liderar estas transformaciones, aunque también se detectan investigadores iberoamericanos que transitan similares derroteros (Rovetta Cortés, 2017; Gómez Espino, 2012). Se inclinan por desarrollar técnicas especiales para niños o adaptar herramientas convencionales al perfil cognitivo y expresivo de la población infantil.

Este enfoque enfrenta a los investigadores con un problema no menor: ¿cuáles son las herramientas más apropiadas para cosechar la perspectiva infantil? Existen numerosas herramientas de carácter narrativo (por ejemplo, Medina, Domínguez y Sánchez Romero, 2013), o para una perspectiva autoevaluativa innovadora (Cubero, Ibarra-Sáez y Rodríguez-Gómez, 2018). Son herramientas, en todo caso, para emplear con jóvenes (generalmente universitarios).

Cabe añadir que desde sus orígenes las técnicas narrativas y proyectivas han sido objeto de controversia en torno a su validez y confiabilidad. Bien recientemente algunos trabajos con base conductista y en la corriente de la neurociencia defienden incluso que no hay un inconsciente para sondear y que, en definitiva, se trata de un mito (Chater, 2018). En la búsqueda tanto de aprovechar un enorme depósito de investigación de campo como de responder a las dudas metodológicas, algunos autores proponen ir hacia la síntesis de ambos campos, la neurología y el psicoanálisis, con estudios empíricos sólidos y apropiados desde el punto de vista epistemológico (García de Frutos, 2011).

Múltiples investigadores utilizan las técnicas narrativas y proyectivas. Son instrumentos mediatizadores que constituyen una vía, a veces la única, para acceder a diferentes estratos de la subjetividad. Convendrá por tanto afinar la forma en que se construye conocimiento. Debe advertirse que estos instrumentos dan 
cuenta de manifestaciones del psiquismo tanto conscientes como inconscientes: fantasías, deseos, ansiedades, expectativas, conflictos y defensas (Sneiderman, 2006).

El valor de las técnicas proyectivas reside en el concepto de "hipótesis puente", ya que serían un enlace entre un conjunto consistente de conceptos que conforman un marco teórico y los observables. Esto constituye una aproximación a la propuesta de síntesis antes indicada. El observable es el dato objetivo: lo dicho o no dicho por un sujeto, su silencio, una pausa, una interrupción en un discurso, un olvido; también las producciones gráficas, los juegos o las respuestas verbales a una técnica propuesta (Sneiderman, 2011).

Un primer nivel de abstracción tras el trabajo de campo es el de las inferencias, que Luciane L'Abate (1967) define como eslabón verbal, formando parte de una categoría mayor de conceptos que representan un proceso de condensación. La inferencia, siendo lo más próximo a lo descriptivo y a lo fenoménico, es asimismo un enlace entre el observable y el supuesto hipotético. La interpretación del material proyectivo constituye un trabajo abductivo: solamente sugiere que algo puede ser, incorporado el carácter transitorio e inestable de las explicaciones de los fenómenos sociales y psicológicos. Esta teoría provisoria consiste en proponer modelos plausibles que deben ser legitimados mediante coherencia explicativa (Sneiderman, 2011).

Volviendo al tema de los observables, con sus respectivos indicadores, que se recolectan con las técnicas, es preciso advertir que su valor está supeditado a la relación que se establece con los demás datos. Entre la variable y el indicador existe apenas una relación de probabilidad, por lo cual debe procurarse que una conjetura o hipótesis derive de múltiples indicadores. Es necesario que el significado de segundo nivel insista a partir de más de una fuente (recurrencias/convergencias). Finalmente, debe tomarse en cuenta que las conclusiones siempre conservan su carácter hipotético, a la vez que son sometidas a tres tipos de exigencia: "1) la evidencia empírica -recurrencias y convergencias (contrastación); 2) la argumentación racional (justificación); 3) la articulación teórica (explicación y comprensión)" (Passalacqua y Febbraio, 2013, p. 52).

Ante el reconocimiento de la falta de precisión de estas técnicas, Didier Anzieu (1981) defiende la necesidad de procurar el acuerdo o confiabilidad inter-jueces, que supone la competencia y el grado de experiencia de los jueces. Testimonio de ello es el relato de los avatares de un ejercicio de interpretación de un Cuestionario Desiderativo, en el que participan 10 jueces. Los porcentajes de acuerdo son muy altos y las diferencias detectadas interesantes. A la luz de estos resultados se concluye que en la confiabilidad inter jueces o en la búsqueda de consenso, la experiencia profesional y el conocimiento de la teoría de base influye notablemente a la hora de interpretar el material de la técnica (Sneiderman, 2011).

Se valoran aquí dos ejemplos provenientes de una investigación ya concluida con niños, se describen las estrategias empleadas y se analizan los resultados obtenidos. Con ocasión de explorar el impacto producido por una propuesta de saturación 
tecnológica en las escuelas públicas uruguayas (modelo 1 a 1), se adopta una entrevista mediatizada por el dispositivo tecnológico entregado a los niños, la entrevista con XO, y se crea una técnica proyectiva, TIC-TAT (Kachinovsky et al., 2013).

Para construir TIC-TAT se tuvieron que atender aspectos epistemológicos, comunicativos y éticos que se valoran a continuación.

\section{Aspectos epistemológicos y metodológicos en investigación cualitativa con niños}

La realidad se construye, y en la construcción de la realidad a la que en este artículo se aboca, deben contemplarse tanto las representaciones de los adultos como las de los niños, sin privilegiar unas sobre otras. Es imposible desligar la subjetividad del investigador de los efectos que esta subjetividad produce con sus intervenciones o indagaciones. No obstante, en la investigación con niños el investigador debe tomar especiales recaudos para no inducir la respuesta esperada (políticamente correcta) y para no interponer su visión adulta frente a los datos recabados. Estos imperativos no responden únicamente a una asimetría intelectual, sino al juego de relaciones de poder desventajosas entre adulto y niño, para quien la aceptación del semejante adulto resulta biológica y psíquicamente imprescindible.

Desde el punto de vista metodológico la investigación cualitativa supone un proceso en el que se definen y redefinen en forma constante las estrategias procedimentales. Los instrumentos privilegiados por esta orientación constructivo-interpretativo son los sistemas conversacionales que, más allá de simples datos, producen una urdimbre de información (González Rey, 2006). Cabe reiterar que, cuando se abordan tópicos de infancia, estos sistemas conversacionales deben nutrirse de la confluencia del discurso adulto e infantil, aspecto que será retomado más adelante.

\section{Modalidades comunicacionales de adultos y niños}

La palabra constituye un componente destacado del discurso infantil, acompañada y articulada al movimiento, al gesto, a la dramatización y al juego (Casas de Pereda, 1991, 1999). Por ello, estas particularidades de la comunicación infantil obligan a reconocer que las entrevistas, cuestionarios o encuestas -técnicas clásicas de la investigación cualitativa- no suelen resultar herramientas oportunas y mucho menos atractivas para los niños, a lo que se podrían agregar limitaciones propias del desarrollo de orden cognitivo. Las características del discurso infantil (producción del dato) y la interpretación de lo comunicado por los niños (significación/construcción del dato) constituyen una fuente de dificultades. Es necesario contemplar las diferentes modalidades expresivas de este discurso singular (acto, gesto, juego, dibujo y palabra) así como los límites de las 
competencias lingüísticas de los niños para comunicar sus ideas o para alcanzar la inteligibilidad de un problema. Algunos tópicos demandan niveles de abstracción y conceptualización aún no logrados, ya sea por factores madurativos o por el tipo de códigos lingüísticos de las micro culturas de pertenencia. Por el motivo que sea, lo figurativo constituye una vía excelente de expresión cuando de niños se trata.

Aunque el énfasis de las indagaciones sobre los problemas de infancia pueda estar puesto en la participación de los niños, ello no implica descartar la mirada de otros protagonistas. La palabra de los padres, docentes, agentes de salud o bienestar social y autoridades, entre otros, suele incluirse con fines de complementariedad o de triangulación metodológica. La cuestión por deliberar es si la palabra de los adultos se considera fuente u obstáculo para la interpretación de datos (Ianni, Kaplan y Failla, 2001), tomando en cuenta las relaciones de poder preexistentes. No es novedoso el efecto de interferencia que tienen los pronunciamientos de los mayores sobre el pensamiento infantil, pero el conocimiento y análisis de dichos pronunciamientos permite evaluar el grado de ajenidad o apropiación de las comunicaciones infantiles obtenidas. Las ciencias sociales, desde diversos recintos disciplinares, se han ocupado en demostrar la inmanencia de lo infantil en lo adulto y de lo adulto en lo infantil. Así cobran realce las revelaciones sobre la "juvenilización" de la especie, es decir, la conservación de rasgos juveniles en el hombre después de haber llegado a la adultez (Morin, 1992).

El progresivo consenso académico y social sobre un decir infantil matrizado por el discurso adulto ha derivado en un creciente cuestionamiento a la veracidad de los testimonios de los niños, nuevo motivo de descalificación. ¿Por qué no otorgar el mismo grado de credibilidad que a los adultos, sin dejar de considerar en unos y en otros la polifonía de voces que anida en la verdad humana?

El aporte de una perspectiva lingüística consiste en revelar que las teorizaciones infantiles o adultas que la investigación cualitativa pretende recabar son sustentadas por medio de una operación discursiva, la argumentación. Los géneros lingüísticos sufren una progresiva complejización, siendo la escolarización un momento clave de este proceso, caracterizado por el fuerte componente ideológico de las estrategias argumentativas. Los niños muestran una marcada inclinación a reproducir el discurso hegemónico escolar (adulto), usando lugares comunes propios de las prácticas institucionales (Román, 2014).

Ante la pregunta por la importancia del discurso adulto, es preciso reafirmar la necesidad de integrar las diferentes perspectivas cuando se procura indagar problemáticas de infancia. No se trata de discursos excluyentes ni complementarios. Cada uno tiene una especificidad a contemplar, por lo que resulta imprescindible contrastar las versiones con sus convergencias y divergencias, tolerando contradicciones que no requieren ser resueltas, ya que dan cuenta de la complejidad a sostener por el investigador. Esa urdimbre de información que se puede construir a partir de los sistemas conversacionales requiere una severa vigilancia del investigador frente a la frecuente tendencia a establecer jerarquías recostadas en diferencias de edad. Es posible concluir que el 
discurso adulto no es fuente ni obstáculo; y, por otra parte, la voz del niño es sólo una parte de lo que permite construir lo que refleja una realidad.

\section{Derechos de infancia que condicionan una particular ética de la investigación}

La investigación con niños obliga a replantear cuestiones éticas y legales, utilizadas a veces como excusas para prescindir de la participación infantil. El reconocimiento de los derechos de infancia y la reconceptualización de los niños como agentes activos, no como meros objetos de investigación, ha promovido una revisión de la ética de investigación con niños. Cualquier proyecto con seres humanos debe velar por el cumplimiento de tres principios básicos: respeto a las personas, beneficencia y justicia (Código de Nüremberg, 1946) (http://bioeticanet.info). Ahora bien, "si la renuncia a la falsedad y a la manipulación de los informantes y de la información para la búsqueda de un conocimiento completo de la realidad constituyen las bases éticas de cualquier investigador, la investigación con niños/as requiere adicionales salvaguardias" (Gómez Espino, 2012, p. 48). A ellas se va a hacer alusión, al examinar los principios del Código de Nüremberg.

El primero de estos principios supone considerar a las personas como seres autónomos, con poder de decisión, así como proteger a los que tienen su autonomía disminuida. Tal es el caso de los niños. Para cumplir con este principio es imprescindible que participen voluntariamente, contando con información adecuada. Las acciones previstas incluyen la solicitud de consentimiento informado a padres o sustitutos y el asentimiento informado de los menores.

El miramiento por la confidencialidad supone la privacidad de los datos proporcionados y de la identidad de los niños, así como el cuidado de su integridad psíquica, física y moral. Existen, sin embargo, límites a la confidencialidad. Ésta no puede ser garantizada si ello supone cometer omisión de asistencia, permitir un daño a terceros o encubrir transgresiones morales y legales. Conviene comunicar al niño que, si es preciso compartir algo de lo que diga, será conversado previamente con él.

El principio de beneficencia postula que tratar éticamente a las personas implica respetar sus decisiones, protegerlas de daños y procurar su bienestar, lo cual entraña dos reglas básicas: no causar daños o minimizar aquellas incomodidades imprevistas y acrecentar al máximo los beneficios. Se impone además realizar instancias de comunicación de los resultados a todos los sujetos participantes (niños, padres, educadores). Por último, y no menor por ello, el principio de justicia propende a considerar modos ecuánimes de distribuir costos y utilidades, prestando atención a la selección de la muestra de investigación. Se pretende que los resultados beneficien tanto a los sujetos involucrados como a la totalidad de la población. Deben tomarse especiales recaudos frente al fenómeno de estigmatización de los niños elegidos para formar parte de la muestra. Frente a ello, es forzoso trabajar y orientar al cuerpo docente sobre cómo trasmitir los 
objetivos de la investigación y los criterios de selección empleados, amén de hacerlos explícitos en el primer contacto con cada niño.

\section{Método}

La finalidad del estudio ha sido valorar la importancia de reconocer a los niños en su calidad de informantes calificados para, con ello, dar cuenta de problemáticas que los afectan directamente, por medio de técnicas específicas de recolección de datos que a veces hay que diseñar expresamente. El desafío asumido es el de aportar herramientas apropiadas, describiendo estrategias de sus investigadores al introducir posibles modos de interrogar la percepción subjetiva de algunos escolares (opiniones y significados atribuidos) sobre el uso e impacto de las TIC en sus vidas y en su entorno. Y para ello se pretende valorar su pertinencia para recabar la perspectiva infantil.

Este estudio se basa en una investigación ya concluida, cuyo objetivo fue explorar los efectos producidos por el Plan Ceibal, proyecto socioeducativo basado en una propuesta de saturación tecnológica implantada en las escuelas públicas uruguayas. La computadora portátil entregada con este propósito a niños y maestros sin excepción ha sido denominada "Ceibalita" o "XO". En la investigación más amplia a la que pertenece este estudio se trabajó con casos en distintos países de América Latina y España. En concreto, por mor del tiempo asignado en la financiación pública del estudio y los recursos disponibles para la investigación, se desarrollaron dos estudios de caso en otros tantos centros educativos de educación primaria de cada país. Se seleccionaron escuelas con gran involucramiento en la integración de las TIC en el aula y que cumplieran los requisitos de estar ubicadas en entornos desfavorecidos tanto rurales como urbanos. Se contó con el apoyo de informantes clave en cada sistema educativo. En cada uno de los centros se entrevistó a directivos, maestros y padres, se aplicó un cuestionario sobre las características de la docencia con Ceibalitas a los maestros, se hizo observación del trabajo en aula y se aplicaron seis entrevistas con XO y técnicas TIC-TAT a otros tantos escolares. Para seleccionarlos se buscó a aquellos estudiantes que, a juicio de los maestros, tuvieran una situación familiar más desfavorecida, y se intentó que hubiera un equilibrio de género en las entrevistas sostenidas.

Como instrumentos, se han utilizado dos herramientas: entrevista con XO y TIC-TAT. Con respecto a la entrevista con XO, se trata de una herramienta mediatizada por el dispositivo tecnológico entregado a los niños, e inspirada en una entrevista "de juego". El juego debe ser considerado un análogo del discurso adulto. Desde hace algunas décadas se utiliza esta modalidad expresiva en diversos ámbitos como estrategia fundamental para el trabajo con niños (Klein, 1955; Aberastury, 1984; Freire de Garbarino, 2017). En esta ocasión la tradicional caja de juegos fue sustituida por la XO, proporcionando buenos resultados dentro de lo que podría considerarse un equivalente de la entrevista en profundidad, 
habitualmente usada con jóvenes y adultos. Con la intención de indagar el nivel de apropiación de la herramienta por los escolares beneficiados y sus preferencias de uso (aplicaciones), se les pedía a los entrevistados que mostraran qué hacían en sus "laptops" y que explicaran cómo lo hacían. El investigador intercalaba preguntas entre sus demostraciones. La información recabada por esta estrategia de exploración podría triangularse con otra, que consiste en el análisis del diario digital. Esta técnica del diario se ha utilizado con motivo de otro estudio en escuelas españolas (Sánchez-Antolín, Andrés y Paredes-Labra, 2018), esta vez sobre la competencia digital facilitada por los programas de saturación tecnológica, donde se han utilizado como técnicas indirectas de sondeo el diario digital de estudiantes de 12 años al final de la etapa primaria. Siempre supeditado a su consentimiento, se accede así a un registro objetivo de la actividad desarrollada con computadoras y otros dispositivos digitales a lo largo del día.

El tiempo requerido por la entrevista con XO y la necesidad metodológica de atender los criterios de la muestra obligó a los investigadores a buscar otras técnicas complementarias, que además habilitaran la triangulación de datos, como luego se dirá con más detalle. La técnica por componer debía ser poco sensible a los discursos sociales imperantes, copiosos y exaltados en ese momento por lo novedoso de la incorporación de tecnologías a las escuelas, también por la inversión del país y las expectativas comprometidas. En otro lugar se ha declarado que desvelaba a los investigadores la idea de aproximarse a la verdad del estudiante y se entendía que, para ello, se necesitaba una técnica que utilizara una vía indirecta de interrogación que, a la vez, atenuara los controles defensivos (Kachinovsky et al., 2013). Se creía, asimismo, que la verdad buscada abarcaría las emociones que envolvían la experiencia con Plan Ceibal, es decir, las vivencias infantiles sobre la inclusión de la XO en la escuela y en sus vidas. Lejos de la pretensión de arribar a una profunda especulación filosófica sobre la verdad, se procuraba la "verdad narrativa".

Se creó entonces el TIC-TAT, una técnica proyectiva inspirada en el TAT, Test de Apercepción Temática (Murray, 1979), en la que se utilizan láminas con estímulos visuales ambiguos para promover la narración de historias. Las técnicas proyectivas están avaladas en la práctica investigadora con enfoques cualitativos, o contribuyendo a enfoques mixtos, tanto para facilitar la expresión no verbal, cuando conviene recoger pensamientos, percepciones, sentimientos y necesidades de los participantes en un estudio, como para hablar de todo ello. Pueden ser cuestiones no sometidas a racionalización por los sujetos o temas problemáticos o no aceptados socialmente. Pueden estar relacionadas con prácticas sociales de difícil observación, como el aprecio y el consumo de determinados productos. Permiten generar relatos o narrativas que pueden ser analizadas. Se han documentado muy diversos procedimientos: dramatización, música, dibujos, fotoelicitaciones, construcción de collages y generación de secuencias de imágenes o "storyboards", entre otros. Lo importante no son tanto las producciones como las explicaciones que ofrecen sus autores (Given, 2008). Aun tomando en cuenta la 
subjetividad de las mismas, se las concibe equivalentes a técnicas tales como las entrevistas en profundidad, para estudios cualitativos, y a inventarios estandarizados en el campo experimental (Hernández Sampieri, Fernández Collado y Baptista, 2010).

Se pasa a describir la técnica. En la primera de las láminas utilizadas en TICTAT, el sexo del único personaje, la acción realizada y el supuesto objeto en sus manos poseen un grado importante de indefinición; puede percibirse allí una laptop, un libro u otro objeto cualquiera (figura 1). Tanto esta lámina como la siguiente fueron generadas deliberadamente con muy baja calidad. Se ha tratado adicionalmente de garantizar los derechos de los niños participantes añadiendo cierta anonimización al ocultar sus caras.

\section{Figura 1}

Tic-Tat. Lámina 1. Elaboración propia

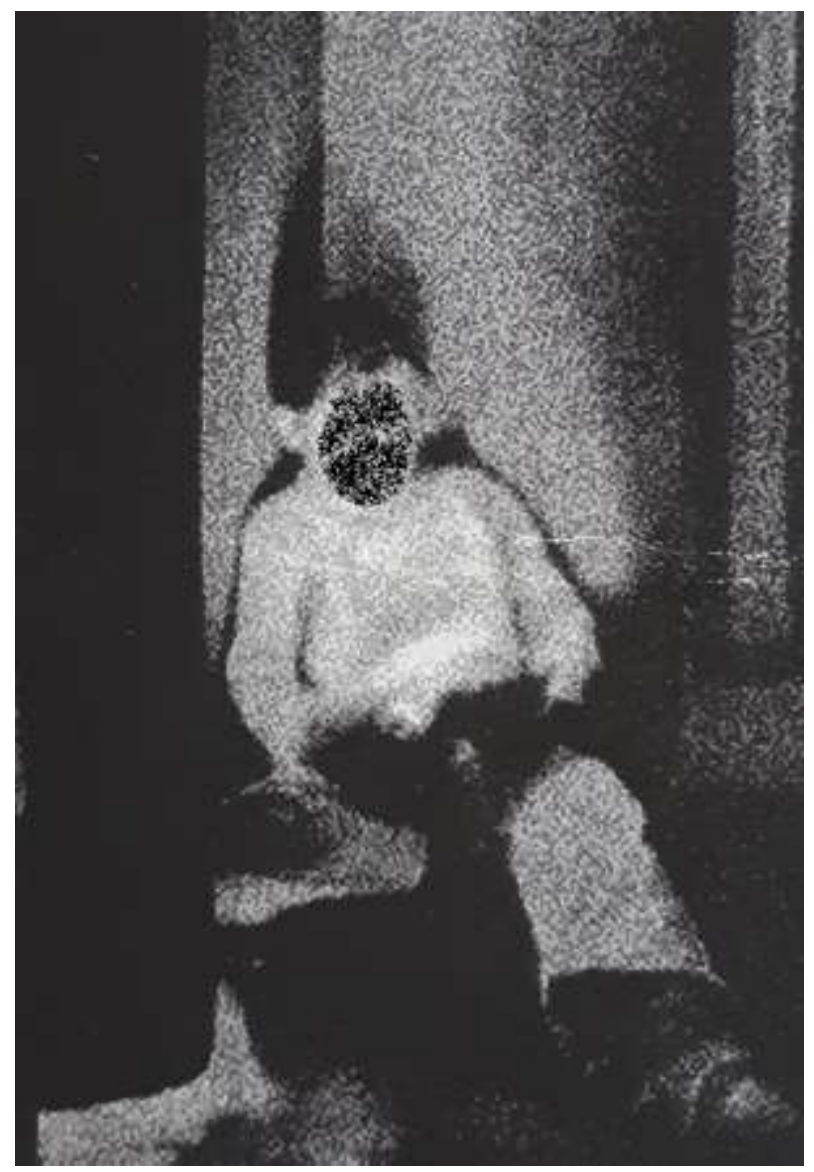




\section{Figura 2}

\section{Tic-Tat. Lámina 1. Elaboración propia}

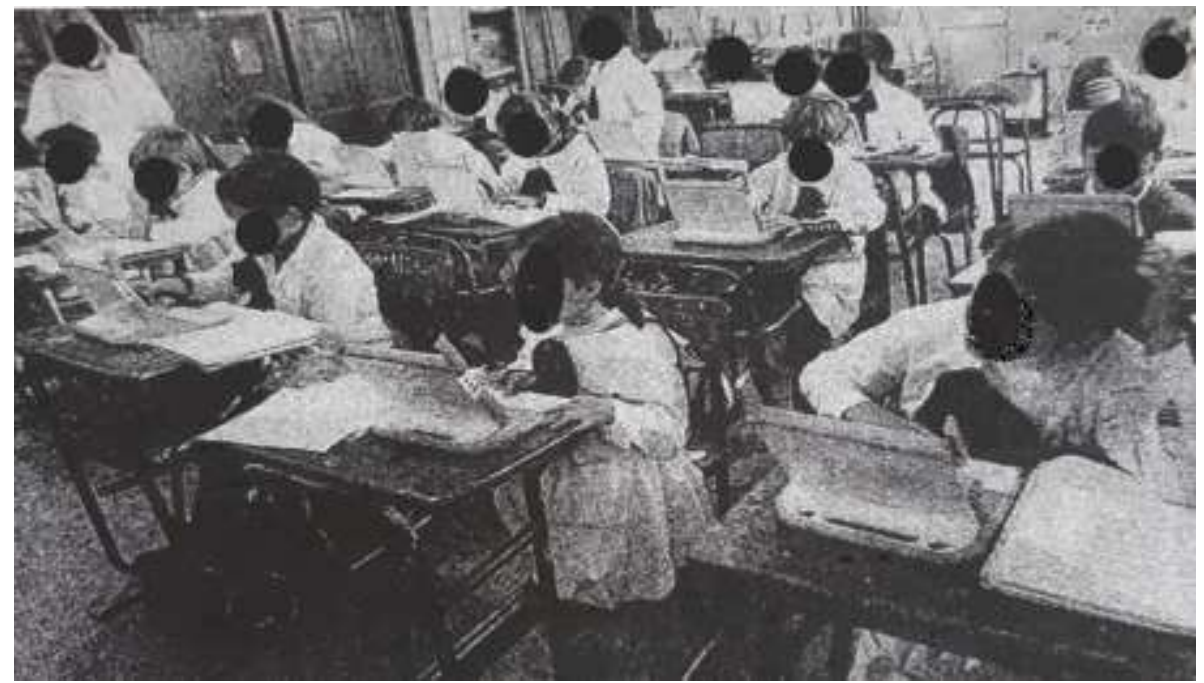

La cuarta lámina (figura 2) resulta también ilustrativa por sus diferencias con el ejemplo precedente. En ella, la XO sí es un objeto definido, ubicado en un contexto de aula en el que se divisa con claridad la figura docente. El aprendizaje escolar constituye una actividad central de la escena, y la ceibalita oficia como potencial mediador del conocimiento. Aunque la mayoría de los personajes lucen ocupados con la laptop, se observa un niño que parece deambular por el aula y otro que observa la labor de su compañero, para copiarle o consultarle.

Se hizo una validación de expertos. Se buscaron "voces cualificadas" (Hernández Sampieri, Fernández Collado y Baptista, 2010), un grupo de seis investigadores de los campos de la tecnología educativa, la educación y la psicología del desarrollo. Para ello, se les sometió el escenario que se generaría, las láminas y el listado de preguntas posibles a consideración. Con sus valoraciones, se mejoró la formulación de los interrogantes.

Las técnicas se pusieron en práctica en las distintas escuelas a lo largo de la primavera de 2016. A partir de la peculiar interpretación que el niño hace de cada una de las escenas ofrecidas es posible inferir expectativas, temores, deseos y conflictos en los que se anudan la particularidad de la imagen concreta con la subjetividad de quien la percibe.

El concepto de proyección justifica y fundamenta la noción de técnica proyectiva, siendo un término que comporta diversas acepciones en virtud de la extensión de su uso. En sentido amplio, la proyección es concebida como una operación del psiquismo por medio de la cual el sujeto localiza en el otro (persona o cosa) cualidades, sentimientos, deseos, que puede no reconocer o rechazar en sí 
mismo. Freud recurrió a este concepto para explicar diversas manifestaciones de la psicología normal y patológica, pero insistió en el carácter normal del mecanismo (Laplanche y Pontalis, 1981). En la base de las pruebas proyectivas se encuentra la idea de ofrecer al sujeto estímulos indeterminados o poco estructurados para que los identifique y estructure de acuerdo con sus temores, conflictos y deseos. Se entiende que el sujeto percibe y responde al medio ambiente según sus propios intereses, aptitudes, hábitos y estados afectivos, duraderos o momentáneos.

Un concepto solidario con las afirmaciones anteriores es el de apercepción, que toma como punto de partida que cualquier percepción del mundo exterior está modalizada por los recuerdos personales, conscientes o inconscientes, y el nivel de ansiedad del sujeto (Anzieu, 1961). La apercepción es una interpretación que da sentido a la experiencia y, como tal, es subjetiva. La percepción de un sujeto se encuentra coloreada por su personalidad, lo cual acarrea una relativa distorsión. La percepción pura y objetiva no existe, siempre es una interpretación singular de la realidad según variables histórico-vivenciales.

No obstante, el sujeto suele no ser consciente del carácter subjetivo de las significaciones atribuidas, ya se trate de describir lo que ocurre en una escena dibujada, de comprender una historia leída o narrada por otro o de construir un relato real o imaginario.

Las entrevistas fueron grabadas y volcadas a un texto. Se realizó un etiquetado con categorías emergentes que fueron refinadas mediante la comparación constante (Flick, 2009).

Evitar el sesgo de confirmación en la interpretación es fundamental. Es un problema epistemológico, que está en la base de la construcción del conocimiento, y también en la evaluación de la misma investigación realizada.

Otros aspectos que refuerzan el rigor del procedimiento de la investigación son: la forma de selección de participantes, las evidencias aportadas, la teoría que guía el análisis, la forma de generar categorías, el manejo de discrepancias entre datos y la presencia de dificultades para construir el análisis (Flick, 2009).

Para evitar el sesgo, una importante herramienta es la triangulación reflexiva (Buetow, 2019), que supone conseguir distintas evidencias con idea de poder reconstruir una situación de forma intersubjetiva. Se debe realizar en la mayor cantidad de sus formas que sea posible: metodológica, de participantes implicados, de investigadores participantes, espacial y temporal (Denzin, 1989; Flick, 2009). A tal fin, en los estudios descritos hubo variedad de evidencias (documentales, observacionales, entrevistas), de participantes (profesores, directivos, padres, estudiantes), investigadores implicados en la realización de entrevistas y lectores de los informes y escuelas implicadas. No se pudo realizar el estudio en diferentes cursos, por limitaciones presupuestarias y tipo de convocatoria que lo facilitó.

De manera adicional, procurando la fiabilidad de la técnica y la credibilidad de los datos (Flick, 2009), fueron devueltos a algunos participantes. En ese sentido, los resultados de las entrevistas fueron volcados a informes que se compartieron 
con las familias y fueron explicados en su lenguaje a los niños, lo que ofreció resonancias que mejoraron estos informes.

Ahora bien, el objeto de este estudio fue, en primer lugar, valorar la competencia de la prueba TIC-TAT para alcanzar el efecto esperado (producción discursiva espontánea y autoría de pensamiento) y explorar lo que se propone (concordancia y especificidad de las respuestas brindadas respecto a las interrogantes o tareas planteadas). Para ello se elaboró, a partir de la revisión de literatura sobre la dimensión epistemológica, comunicativa y ética, una lista de control sobre la prueba. En esta lista de control se trató de evaluar por parte de los investigadores del equipo internacional la pertinencia de las respuestas proporcionadas, la iniciativa de las conversaciones, la fluidez de las mismas, el compromiso con la actividad, la autoría del pensamiento, la reflexividad y la aparición de pensamiento crítico en las entrevistas mantenidas.

Conviene indicar limitaciones de esta investigación. Como en todo estudio cualitativo, conviene advertir que, con estas investigaciones, se trata más de "comprender" un fenómeno (los usos que hacen los niños de la tecnología) que de "explicar"; no se buscan, por tanto, causalidades a esos usos.

Además, los estudios se han realizado en un contexto latinoamericano de saturación tecnológica, con una selección de centros educativos y por un tiempo limitado.

Como ocurre con los estudios de caso, serán sucesivos estudios los que, habiendo examinado cuidadosa y reflexivamente las condiciones en que se desarrollaron estos, ofrezcan resonancias (conexiones temáticas y de otra índole con la propia realidad) y sugieran realizar nuevos análisis a otros investigadores.

\section{Resultados}

Se realizó el análisis de la prueba TIC-TAT con arreglo a la lista de control definida sobre la revisión de literatura.

Con ello fue posible caracterizar la producción infantil. Se observó que las respuestas proporcionadas por los niños eran pertinentes. Esto era así porque los estudiantes responden a lo que se les pregunta. Ellos llevaron la iniciativa conversacional. Con frecuencia introdujeron tópicos no presentados por los investigadores y propusieron categorías emergentes. Hubo fluidez conversacional. Denotaron espontaneidad o naturalidad en la formulación de sus consideraciones, gestos o acciones. También se observó compromiso con la actividad, esto es, manifestaron preocupación por la adecuación, claridad o precisión de sus respuestas. Por otra parte dieron muestra de autoría de pensamiento, es decir, de autonomía intelectual, no de sometimiento a posibles expectativas de sus referentes adultos o investigadores. Hubo reflexividad, es decir, usaron de manera adecuada el género argumentativo. Y, para terminar, se observó el advenimiento de un pensamiento crítico, se cuestionaron lo canónico o instituido cuando lo entendieron necesario. 
La aplicación de la técnica del TIC-TAT ha supuesto, en la investigación sobre Plan Ceibal, un facilitador para el despliegue de una narrativa infantil rebosante de autenticidad. Permitió deconstruir e impugnar dos antinomias presentes en el discurso adulto sobre las consecuencias de la disponibilidad de las computadoras entregadas a los niños (niño propietario), antinomias que han sido sintetizadas como "tecnología vs. vínculo" y “juego vs. aprendizaje” (Kachinovsky et al., 2013). En cuanto a la primera antinomia, la importancia del otro humano no dejaba de ser subrayada por los niños, ya sea como alegato a su presencia o como reprobación de su ausencia. En cuanto a la segunda, el uso de la tecnología con fines de aprendizaje fue asimismo destacado, aunque coexistiera con su utilización con fines lúdicos (esperable por ser niños).

De forma global, al valorar la idoneidad de estas técnicas para concitar el punto de vista infantil se observa que la recolección de datos a través de la técnica del TIC-TAT recurre a estímulos visuales para convocar la voz de los niños. Desde el punto de vista de los fenómenos perceptivos, la imagen hace presente lo que representa, circunstancia que se corresponde con los tiempos semióticos de la simbolización en estos primeros años (Casas de Pereda, 1999). Es por tal motivo que resultan herramientas idóneas para suscitar la palabra infantil.

Se observa, por otra parte, que esta técnica recurre al mecanismo psíquico de la proyección, mediante el cual es posible tratar temas conflictivos desplazados a otros objetos alejados de la persona propia. Se minimizan las ansiedades en juego, se protege la intimidad del niño y se potencia la productividad verbal referida a la percepción subjetiva del problema objeto de investigación.

\section{Discusión y conclusiones}

En este trabajo se ha cuestionado la frecuente ausencia del punto de vista de los niños como informantes calificados en investigación cualitativa sobre temas y problemas de infancia.

A lo largo del trabajo se ahonda en diferentes problemas implicados de manera directa en el desarrollo de la investigación llevada a cabo. El primero de ellos, imprescindible de abordar, es la producción infantil de narrativa sobre el uso de las computadoras. Respecto a este punto, para el trabajo que se ha desarrollado, era necesario poder constatar el nivel de narrativa sobre computadoras de los estudiantes. Partiendo de la revisión teórica realizada, se observa que no existe una forma de hacerlo en el ámbito educativo. Así, se ha procedido al diseño y propuesta de un instrumento de medición dando respuesta y abordando las necesidades que este trabajo planteaba.

Los resultados obtenidos en este proceso muestran que la prueba elaborada para estudiantes de último curso de educación primaria ofrece posibilidades de producción narrativa adecuadas, así como de fiabilidad de dichas narrativas, al ser triangulados sus resultados con otras evidencias, con lo que puede ser utilizada para la finalidad descrita. 
Se destaca la calidad del discurso generado por los estudiantes, concordando las respuestas que dan en TIC-TAT con lo que plantean en otras técnicas, y reduciendo aspectos ansiógenos, algo en cierto modo inesperado en la literatura consultada. En ese sentido, las respuestas facilitadas han sido más estables que en la técnica TAT original (Bernstein, 1979). Es posible que las características de la muestra hayan influido en este tipo de respuestas, por lo que queda sujeto a sucesivos estudios posteriores.

Por otra parte, en los resultados aparecen los temas del adulto mediador y el juego. Con respecto a los adultos, se pone de manifiesto, entonces, su importancia (educadores, padres) cuando los estudiantes utilizan las computadoras, lo que es consistente con la literatura sobre el uso de medios y que recoge alguna investigación reciente que se pregunta por la relación de los padres con la escuela a través de computadores (Freitas-Cortina, Paredes-Labra y Sánchez-Antolín, 2019).

Con respecto a vivencia lúdica o de juego en el trabajo con computadoras en clase, se abre una oportunidad indirecta para recomendar el uso de metodologías de este tenor, pues son esperadas por los estudiantes, si bien esta temática escapa a los objetivos del estudio.

En su conjunto parece logrado el objetivo del estudio, que era el de incluir a los niños en calidad de informantes calificados mediante técnicas específicas (entrevistas con computadora y técnica TIC-TAT).

Se ha valorado y descrito la puesta en práctica de técnicas que han resultado apropiadas para investigar las repercusiones del uso generalizado de las TIC con escolares. Dichas técnicas permiten inferir orientaciones generales para su aplicación y ulterior desarrollo de herramientas similares, tales como recurrir a estímulos visuales en distintos soportes (fotos, dibujos, historietas, entre otros) con el objetivo de estimular la narrativa infantil.

Cabe destacar la pertinencia de estas técnicas cuando se pone en juego la investigación con niños, en la medida que permite acceder a una vía indirecta de interrogación que, a la vez, rebaja los controles defensivos y atenúa la fuerza de lo canónico y el discurso adulto imperante.

\section{Referencias}

Aberastury, A. (1984). Teoría y técnica del psicoanálisis de niños. Buenos Aires: Paidós.

Anzieu, D. (1961). Los métodos proyectivos. Buenos Aires: Ábaco.

Bernstein, J. (1979). El test proyectivo de Murray. En: Murray, H., Test de Apercepción Temática (TAT), pp.13-18). Buenos Aires: Paidós.

Buetow, S. (2019). Apophenia, unconscious bias and reflexivity in nursing qualitative research. International Journal of Nursing Studies, (89), 8-13.

Casas de Pereda, M. (1991). Gesto, juego y palabra. El discurso infantil. Revista 
Uruguaya de Psicoanálisis, (74), 25-44.

Casas de Pereda, M (1999). En el camino de la simbolización: producción del sujeto psíquico. Buenos Aires: Paidós.

Chater, N. (2018). The mind is flat. Londres: Penguin.

Denzin, N.K. (1989) The Research Act. $3^{a}$ ed. Englewood Cliffs: Prentice Hall.

Flick, U. (2009). An introduction to qualitative research. 4⿳a ed. Londres: Sage.

Freire de Garbarino, M. (2017). La entrevista de juego. Revista Uruguaya de Psicoanálisis, (124), 137-173.

Freitas-Cortina, A.; Paredes-Labra, J.; Sánchez-Antolín, P. (2019). Los espacios intermedios de la relación entre familia y escuela en contextos de inmersión tecnológica. Revista latinoamericana de tecnología educativa. (Relatec), 18(1), 41-53.

García de Frutos, H. (2011). Neurociencias y psicoanálisis: consideraciones epistemológicas para una dialéctica posible sobre la subjetividad. Revista de la Asociación Española de Neuropsiquiatría, 31(4), 661-678. https://dx.doi.org/10.4321/S0211-57352011000400005.

Gibbs, G. (2012). El análisis de datos cualitativos en investigación cualitativa. Madrid: Morata.

Given, L. (2008). The SAGE encyclopedia of qualitative research methods. Thousand Oaks: Sage.

Gómez Espino, J. M. (2012). El grupo focal y el uso de viñetas en la investigación con niños. EMPIRIA. Revista de Metodología de las Ciencias Sociales, (24), 45-65.

González Rey, F. (2006). Investigación cualitativa y subjetividad. Guatemala: Oficina del Arzobispado de Derechos Humanos.

Hernández Sampieri, R.; Fernández Collado, C.; Baptista, M.P. (2010). Métodos de investigación. México: McGrawHill

Ianni, G., Kaplan, E., \& Failla, H. (2001). La biografía del niño redactada por los padres: ¿fuente u obstáculo para la interpretación? Psicoanálisis. Revista de la Asociación Psicoanalítica de Buenos Aires, 23(3), 603-613.

Kachinovsky, A., Martínez, S., Gabbiani, B., Gutiérrez, R., Rodríguez Rava, B., Ulriksen, M. y Achard, P. (2013). Impacto del Plan Ceibal en el funcionamiento cognitivo y lingüístico de los niños. En: Rivoir, A. (coord.). Plan Ceibal e inclusión social. Perspectivas interdisciplinarias, 99-155. Montevideo: Plan Ceibal - Universidad de la República.

Kirk, S. (2007). Methodological and ethical issues in conducting qualitative research with children and young people: a literature review. International Journal of Nursing Studies, 44(7), 1250-1260.

Klein, M. (1955). La técnica psicoanalítica del juego: su historia y significado. 
En M. Klein Obras Completas (3). Buenos Aires: Paidós.

L’Abate, L. (1967). Principios de Psicología Clínica. Buenos Aires: Paidós.

Laplanche, J., \& Pontalis, J.-B. (1981). Diccionario de psicoanálisis. Barcelona: Labor.

Medina Rivilla, A., Domínguez Garrido, M.C., \& Sánchez Romero, C. (2013). Evaluación de las competencias de los estudiantes: modelos y técnicas para la valoración. Revista de Investigación Educativa, 31(1), 239-255. http://dx.doi.org/10.6018/rie.31.1.157601

Morin, E. (1992). El paradigma perdido. Barcelona: Kairós.

Murray, H. (1979). Test de apercepción temática (TAT). Manual para la aplicación. Buenos Aires: Paidós.

Passalacqua, A.M. \& Febbraio, A. (2013). Acerca de la validez y confiabilidad de las técnicas proyectivas. $V$ Congreso Internacional de Investigación y Práctica Profesional en Psicología XX Jornadas de Investigación - Noveno Encuentro de Investigadores en Psicología del MERCOSUR. Facultad de Psicología - Universidad de Buenos Aires, Buenos Aires. Disponible en: https://www.aacademica.org/000-054/908

Román, S. (2014). Una aproximación crítica al estudio del discurso argumentativo infantil en el marco de la educación primaria (Tesis de maestría). Universidad de la República, Facultad de Humanidades y Ciencias de la Educación, Montevideo, Uruguay. Recuperado de https://www.colibri.udelar.edu.uy/jspui/handle/123456789/9275.

Rovetta Cortés, A. (2017). Si me dieran un billete de avión...: recurriendo a la elucidación gráfica en entrevistas con menores de edad. EMPIRIA. Revista de Metodología de Ciencias Sociales, (36), 63-87.

Sánchez-Antolín, P., Andrés, C., Paredes-Labra, J. (2018). El papel de la familia en el desarrollo de la competencia digital. Análisis de cuatro casos. Digital Education Review, 34, 44-58.

Sneiderman, S. (2006). Las técnicas proyectivas como método de investigación y diagnóstico. Actualización en técnicas verbales: "El cuestionario desiderativo". Subjetividad y Procesos Cognitivos, 8, 296-331.

Sneiderman, S. (2011). Consideraciones acerca de la confiabilidad y validez en las técnicas proyectivas. Subjetividad y Procesos Cognitivos, 15(2), 93-110. 
